\title{
A new framework for complexity analysis in international development projects - Results from a Delphi study
}

\author{
Gajić, S. ${ }^{a,}{ }^{*}$, Palčič, I. ${ }^{b}$ \\ ${ }^{a}$ University of Novi Sad, Faculty of Technical Sciences, Novi Sad, Serbia \\ ${ }^{b}$ University of Maribor, Faculty of Mechanical Engineering, Maribor, Slovenia
}

\begin{abstract}
A B S T R A C T
The main objective of this paper is to develop a framework for characterising project complexity in International Development (ID) projects. Contemporary challenges in ID projects have led to their growth in their complexity, which in recent years has driven researches in recent years to publish numerous papers that deal with this topic, demonstrating its importance in current project management research. Nevertheless, existing literature lacks in generally accepted framework that considers specifics of project complexity in ID projects. Thus, new framework was developed, based on a two-round Delphi survey, building upon existing TOE (technology-organisation-environment) framework with new empirical insights given from the experts in the field of ID projects. The main contribution of the paper is the validation of existing TOE complexity factors, in the context of International Development projects. Additionally, eight new complexity factors were proposed by the experts, and it was concluded that Environmental complexity had the biggest impact on International Development projects. From a managerial perspective, proposed complexity framework can be used for making a complexity footprint, which could indicate the critical areas of the project where complexity could be expected. In addition, the model represents a novel theoretical lens for assessing complexity in ID projects.
\end{abstract}

2019 CPE, University of Maribor. All rights reserved.

\author{
ARTICLE INFO \\ Keywords: \\ International development pro- \\ jects (ID); \\ Project management; \\ Complexity; \\ Project complexity; \\ Technology-organisation- \\ environment (TOE); \\ Delphi study \\ *Corresponding author: \\ gajic.sladjana@uns.ac.rs \\ (Gajić, S.) \\ Article history: \\ Received 20 December 2018 \\ Revised 15 May 2019 \\ Accepted 20 May 2019
}

\section{References}

[1] Ika, L.A., Söderlund, J., Munro, L.T., Landoni, P. (2018). Special issue: When project management meets international development, what can we learn?, International Journal of Project Management, Vol. 36, No. 2, 331333, doi: 10.1016/j.ijproman.2017.05.005.

[2] Flyvbjerg, B. (2014). What you should know about megaprojects and why: An overview, Project Management Journal, Vol. 45, No. 2, 6-19, doi: 10.1002/pmj.21409.

[3] Ika, L.A., Diallo, A., Thuillier D. (2012). Critical success factors for World Bank projects: An empirical investigation, International Journal of Project Management, Vol. 30, No. 1, 105-116, doi: 10.1016/j.ijproman. 2011.03.005.

[4] Ika, L.A., Hodgson, D. (2014). Learning from international development projects: Blending critical project studies and critical development studies, International Journal of Project Management, Vol. 32, No. 7, 1182-1196, doi: 10.1016/i.ijproman.2014.01.004.

[5] Standish Group, Chaos summary (2009), from https://www.projectsmart.co.uk/white-papers/chaos-report.pdff accessed October 10, 2018.

[6] Brady, T., Davies, A. (2014). Managing structural and dynamic complexity: A tale of two projects, Project Management Journal, Vol. 45, No. 4, 21-38, doi: 10.1002/pmj.21434.

[7] Cicmil, S., Cooke-Davies, T., Crawford, L., Richardson, K.A. (2009). Exploring the complexity of projects: Implications of complexity theory for project management practice, Project Management Institute, Newtown Square, Pennsylvania, USA. 
[8] Geraldi, J., Maylor, H., Williams, T. (2011). Now, let's make it really complex (complicated): A systematic review of the complexities of projects, International Journal of Operations \& Production Management, Vol. 31, No. 9, 966990, doi: $10.1108 / 01443571111165848$.

[9] Vidal, L.-A., Marle, F. (2008). Understanding project complexity: Implications on project management, Kybernetes, Vol. 37, No. 8, 1094-1110, doi: 10.1108/03684920810884928.

[10] Saynisch, M. (2010). Beyond frontiers of traditional project management: An approach to evolutionary, selforganizational principles and the complexity theory - Results of the research program, Project Management Journal., Vol. 41, No. 2, 21-37, doi: 10.1002/pmj.20159.

[11] Kiridena, S., Sense, A. (2016). Profiling project complexity: Insights from complexity science and project management literature, Project Management Journal., Vol. 47, No. 6, 56-74, doi: 10.1177/875697281604700605.

[12] Williamson O.E., Winter, S.G. (1993). The nature of the firm: origins, evolution, and development, Oxford University Press, UK.

[13] Winter, M., Smith, C., Morris, P., Cicmil, S. (2006) Directions for future research in project management: The main findings of a UK government-funded research network, International Journal of Project Management, Vol. 24, No. 8, 638-649, doi: 10.1016/i.ijproman.2006.08.009.

[14] He, Q., Luo, L., Hu, Y., Chan, A.P.C. (2015). Measuring the complexity of mega construction projects in China - A fuzzy analytic network process analysis, International Journal of Project Management, Vol. 33, No. 3, 549-563, doi: 10.1016/j.iproman.2014.07.009.

[15] Geraldi, J., Adlbrecht, G. (2007). On faith, fact, and interaction in projects, IEEE Engineering Management Review, Vol. 36, No. 2, 35-49, doi: 10.1109/emr.2008.4534318.

[16] Cooke-Davies, T., Cicmil, S., Crawford, L., Richardson, K. (2007). We're not in Kansas anymore, Toto: Mapping the strange landscape of complexity theory, and its relationship to project mangement, Project Management Journal, Vol. 38, No. 2, 50-61, doi: 10.1177/875697280703800206.

[17] Bosch-Rekveldt, M., Jongkind, Y., Mooi, H., Bakker, H., Verbraeck, A. (2011). Grasping project complexity in large engineering projects: The TOE (technical, organizational and environmental) framework, International Journal of Project Management, Vol. 29, No. 6, 728-739, doi: 10.1016/j.ijproman.2010.07.008.

[18] Ika, L.A., Donnelly, J. (2017). Success conditions for international development capacity building projects, International Journal of Project Management, Vol. 35, No. 1, 44-63, doi: 10.1016/j.ijproman.2016.10.005.

[19] Golini, R., Landoni, P. (2013). International development projects: Peculiarities and managerial approaches, Project Management Institute, USA.

[20] Cicmil, S., Williams, T., Thomas, J., Hodgson, D. (2006). Rethinking project management: Researching the actuality of projects, International Journal of Project Management, Vol. 24, No. 8, 675-686, doi: 10.1016/ j.ijproman.2006.08.006.

[21] Baccarini, D. (1996). The concept of project complexity - A review, International Journal of Project Management, Vol. 14, No. 4, 201-204, doi: 10.1016/0263-7863(95)00093-3.

[22] Williams, T.M. (1999). The need for new paradigms for complex projects, International Journal of Project Management, Vol. 17, No. 5, 269-273, doi: 10.1016/s0263-7863(98)00047-7.

[23] Sommer, S.C., Loch, C.H. (2004). Selectionism and learning in projects with complexity and unforeseeable uncertainty, Management Science, Vol. 50, No. 10, 1334-1347, doi: 10.1287/mnsc.1040.0274.

[24] Vidal, L.-A., Marle, F., Bocquet, J.-C. (2011). Measuring project complexity using the analytic hierarchy process, International Journal of Project Management, Vol. 29, No. 6, 718-727, doi: 10.1016/j.ijproman.2010.07.005.

[25] Bakhshi, J., Ireland, V., Gorod, A. (2016). Clarifying the project complexity construct: Past, present and future, International Journal of Project Management, Vol. 34, No. 7, 1199-1213, doi: 10.1016/i.ijproman.2016.06.002.

[26] Lu, Y., Luo, L., Wang, H., Le, Y., Shi, Q. (2015). Measurement model of project complexity for large-scale projects from task and organization perspective, International Journal of Project Management, Vol. 33, No. 3, 610-622, doi: 10.1016/j.ijproman.2014.12.005.

[27] Xia, B., Chan, A.P.C. (2012). Measuring complexity for building projects: A Delphi study, Engineering, Construction and Architectural Management, Vol. 19, No. 1, 7-24, doi: 10.1108/09699981211192544.

[28] Lessard, D., Sakhrani, V., Miller, R. (2014). House of project complexity - Understanding complexity in large infrastructure projects, Engineering Project Organization Journal, Vol. 4, No. 4, 170-192, doi: 10.1080/21573727. 2014.907151.

[29] Williams, T. (2003). Modelling complex projects, John Wiley \& Sons, Chichester, UK.

[30] Flick, U. (2018). An introduction to qualitative research, Sixth edition, Sage, London, UK.

[31] Chan, A.P.C., Yung, E.H.K., Lam, P.T.I., Tam, C.M., Cheung, S.O. (2001). Application of Delphi method in selection of procurement systems for construction projects, Construction Management and Economics, Vol. 19, No. 7, 699718, doi: $10.1080 / 01446190110066128$.

[32] Delbecq, A.L., Van de Ven, A.H., Gustafson, D.H. (1975). Group techniques for program planning: A guide to nominal group and Delphi processes, Scott Foresman Company, Glenview, Illinois, USA.

[33] Linstone, H.A., Turoff, M. (2002). The Delphi method: Techniques and applications, Addison-Wesley, USA.

[34] Liu, S., Zhang, J., Keil, M., Chen, T. (2010). Comparing senior executive and project manager perceptions of IT project risk: A Chinese Delphi study, Information Systems Journal, Vol. 20, No. 4, 319-355, doi: 10.1111/j.13652575.2009.00333.x.

[35] Hadaya, P., Cassivi, L., Chalabi, C. (2012). IT project management resources and capabilities: A Delphi study, International Journal of Managing Projects in Business, Vol. 5, No. 2, 216-229, doi: 10.1108/1753837121121 4914.

[36] Perera, B.A.K.S., Rameezdeen, R., Chileshe, N., Hosseini, M.R. (2014). Enhancing the effectiveness of risk management practices in Sri Lankan road construction projects: A Delphi approach, International Journal of Construction Management, Vol. 14, No. 1, 1-14, doi: 10.1080/15623599.2013.875271. 
[37] Lang, T. (1998). An overview of four futures methodologies (Delphi, environmental scanning, issues management and emerging issue analysis), The Manoa Journal of Fried and Half-Fried Ideas (About the future), Hawaii Research Center for Futures Studies, Honolulu, from http://citeseerx.ist.psu.edu/viewdoc/summary ?doi=10.1.1.455.1045, accessed October 8, 2018.

[38] Okoli, C., Pawlowski, S.D. (2004). The Delphi method as a research tool: An example, design considerations and applications, Information \& Management, Vol. 42, No. 1, 15-29, doi: 10.1016/i.im.2003.11.002.

[39] Hallowell, M.R. (2009). Techniques to minimize bias when using the Delphi method to quantify construction safety and health risks, In: Ariaratnam, S.T., Rojas, E.M., (eds.), Building a Sustainable Future, American Society of Civil Engineers, Seattle, Washington, USA, 1489-1498, doi: 10.1061/41020(339)151.

[40] Schmidt, R.C. (1997). Managing Delphi surveys using nonparametric statistical techniques, Decision Sciences, Vol. 28, No. 3, 763-774, doi: 10.1111/i.1540-5915.1997.tb01330.x.

[41] Von der Gracht, H.A. (2012). Consensus measurement in Delphi studies: Review and implications for future quality assurance, Technological Forecasting and Social Change, Vol. 79, No. 8, 1525-1536, doi: 10.1016/ j.techfore.2012.04.013. 


\section{APEM}

\title{
Nov okvir za analizo zahtevnosti v mednarodnih razvojnih projektih - rezultati študije Delphi
}

\author{
Gajić, S. ${ }^{a,}{ }^{*}$, Palčič, I. ${ }^{b}$ \\ ${ }^{a}$ University of Novi Sad, Faculty of Technical Sciences, Novi Sad, Serbia \\ ${ }^{b}$ University of Maribor, Faculty of Mechanical Engineering, Maribor, Slovenia
}

\section{POVZETEK}

Glavni cilj tega prispevka je razviti okvir za opredelitev zahtevnosti mednarodnih razvojnih (ID) projektov. Sodobni izzivi v projektih ID so privedli do njihove večje zahtevnosti, ki je v zadnjih letih raziskovalce spodbudila k objavljanju številnih prispevkov na to temo, kar kaže na njen pomen v trenutnih raziskavah upravljanja projektov. Kljub temu pa v obstoječi literaturi ni zaslediti splošno sprejetega okvira, ki upošteva posebnosti projektne zahtevnosti pri projektih ID. Tako je bil razvit nov okvir, ki temelji na dvokrožni raziskavi Delphi, ki temelji na obstoječem okviru TOE (tehnologija-organizacija-okolje) z novimi empiričnimi spoznanji strokovnjakov s področja ID-projektov. Glavni doprinos prispevka je preverjanje obstoječih dejavnikov zahtevnosti TOE v okviru mednarodnih razvojnih projektov. Poleg tega so strokovnjaki predlagali osem novih dejavnikov zahtevnosti in ugotovili, da okoljska zahtevnost najbolj vpliva na mednarodne razvojne projekte. $\mathrm{Z}$ upravljavskega vidika je mogoče predlagani okvir uporabiti za šablono, ki bi lahko napovedala kritična področja projekta, na katerih bi bilo mogoče pričakovati zahtevnost. Poleg tega model predstavlja novo teoretično sito za oceno zahtevnosti projektov ID.

\section{PODATKI O ČLANKU}

Ključne besede:

Mednarodni razvojni projekti (ID);

Vodenje projektov;

Zahtevnost;

Zahtevnost projekta;

Tehnologija-organizacija-okolje

(TOE);

Študija Delphi

*Kontaktna oseba: gajic.sladjana@uns.ac.rs (Gajić, S.)

Zgodovina članka:

Prejet 20. decembra 2018

Popravljen 15. maja 2019

Sprejet 20. maja 2019 